UDC 517.9

\title{
GLOBAL POSITIONAL SYNTHESIS AND STABILIZATION IN FINITE TIME OF MIMO GENERALIZED TRIANGULAR SYSTEMS BY MEANS OF THE CONTROLLABILITY FUNCTION METHOD
}

\section{ГЛОБАЛЬНИЙ ПОЗИЦЙНИЙ СИНТЕЗ ТА СТАБІЛІЗАЦІЯ В СКІНЧЕННИЙ ЧАС УЗАГАЛЬНЕНИХ ТРИКУТНИХ СИСТЕМ З КІЛЬКОМА ВХОДАМИ ТА ВИХОДАМИ ЗА ДОПОМОГОЮ МЕТОДУ ФУНКЦЇ̈ КЕРОВАНОСТІ}

\author{
V. I. Korobov ${ }^{*}$ \\ Kharkov Nat. Univ. \\ Svobody sqr. 4, Kharkov 61077, Ukraine \\ Inst. Math., Szczecin Univ. \\ Wielkopolska str. 15, Szczecin 70451, Poland \\ e-mail:vkorobov@univer.kharkov.ua \\ korobow@sus.univ.szczecin.pl
}

\section{S. S. Pavlichkov**}

Taurida Nat. Univ.

Vernadsky Av. 4, Simferopol 95007, Ukraine

and Univ. Appl. Sci. Erfurt

Altonaer str. 25, Erfurt 99085, Germany

e-mail: s_s_pavlichkov@yahoo.com

\author{
W. H. Schmidt** \\ Inst. Math. and Inform., Greifswald Univ. \\ Walter-Rathenau str. 47, Greifswald 17487, Germany \\ e-mail: wschmidt@uni-greifswald.de
}

\begin{abstract}
We solve the problem of global stabilization in finite time for a general class of triangular multi-input and multi-output systems with singular input-output links. In order to obtain the main result of the work, we combine the controllability function method (which works locally, around the equilibrium only for our class of triangular forms) with a modification of the global construction developed for the generalized triangular form in the singular case in our previous works.

Розв'язано задачу глобальної стабілізації в скінченний час для загального класу трикутних систем з кількома входами і виходами та сингулярними зв'язками між входом і виходом. Для отримання основного результату поєднано метод функції керованості (що працює в околі нерухомої точки тільки для розглядуваного класу трикутних систем) з модифікацією загальної побудови для узагальненої трикутної форми в сингулярному випадку, що розвинута в попередніх роботах авторів.
\end{abstract}

* The work was partially supported by Polish Ministry of Science and High Education (Grant № N514238438).

** This work was partially supported by DAAD, Germany.

(c) V. I. Korobov, S. S. Pavlichkov, W. H. Schmidt, 2012 
1. Introduction. The problem of feedback stabilization of control systems by means of the Lyapunov second method is a wide area developing extensively and it is hardly possible to give any detailed overview which covers all the aspects.

On the other hand, the problem of stabilization in finite time differs essentially from that of classical Lyapunov stabilization (for instance, because the uniqueness property of the Cauchy problem no longer holds true at the corresponding equilibrium point) which requires new methods. One such constructive method is that of controllability function, which was proposed in [10] and later developed in $[1,11,12]$ and in many other works. In brief, the purport of this approach is that, instead of looking for a Lyapunov function, one constructs the so-called „controllability function" (whose properties are different from those of Lyapunov functions) and finds a feedback to satisfy a certain inequality which can be regarded as an analog of the Lyapunov inequality.

Let us note that (by its construction) the controllability function method has been applied to either linear systems or at least to linearizable ones. During the last decade the problem of stabilization in finite time became a hot topic (see, for example, $[2,7,8,16]$ ), especially in the context of applicability of backstepping designs [7,8].

Therefore, it is natural to raise the problem of ( global) positional synthesis (i.e., that of stabilization in finite time by means of the controllability function method) for some classes of nonlinear control systems which are not feedback linearizable. The first candidate on this way is the triangular form (next called TF) [4-6, 9, 13-15, 17-21, 23-25].

Although the TF was originally introduced in [9] as a class of nonlinear but feedback linearizable systems (and later was treated by most authors in the context of feedback linearization, see for instance $[6,15,21,23])$, in fact, this class is much richer and the triangular systems of ODE are not necessarily feedback linearizable (the so-called ,singular case” $[4,5,18,19,24]$ ). Unfortunately, even for the classical Lyapunov stabilization, most results were obtained for the regular case; as to the singular case, this requires some specific restrictions and/or developing very specific techniques see $[5,18,24,25]$.

The above-mentioned work [18] was devoted to the global Lyapunov stabilization of the socalled ,generalized" TF systems under the only assumption of the regularity of the equilibrium point at which the system should be stabilized (see also related work [14] devoted to the global robust controllability of this class without that assumption). Being motivated by this work as well as by the controllability function method, we want to combine these two approaches and to solve the problem of global positional synthesis for the class of TF considered in [18], i.e., to construct a feedback, which not only stabilizes this class globally, but also steers any initial state into the equilibrium point in finite time (however, such a feedback cannot be of class $C^{1}$ everywhere, because one cannot provide the uniqueness property for the Cauchy problem at the equilibrium point with such a feedback).

2. Preliminaries. For any vectors $\xi=\left[\xi_{1}, \ldots, \xi_{N}\right]$ and $\eta=\left[\eta_{1}, \ldots, \eta_{N}\right]$ in $\mathbf{R}^{N}$ (with arbitrary $N \in \mathbf{N}$ ), and any $R>0$, we use the following notation:

$$
\begin{gathered}
\langle\xi, \eta\rangle:=\sum_{i=1}^{N} \xi_{i} \eta_{i}, \quad|\xi|:=\left(\sum_{i=1}^{N} \xi_{i}^{2}\right)^{\frac{1}{2}}, \quad|\eta|:=\left(\sum_{i=1}^{N} \eta_{i}^{2}\right)^{\frac{1}{2}} ; \\
B_{R}(\xi):=\left\{\eta \in \mathbf{R}^{N}|| \eta-\xi \mid<R\right\} .
\end{gathered}
$$


For any $\Omega \subset \mathbf{R}^{N}$ by $\bar{\Omega}$ we denote its closure.

Our background is the following theorem (see [10] and [12, p. 19]).

Theorem 2.1 [10,12]. Consider the control system

$$
\dot{x}=f(x, u)
$$

with states $x \in \mathbf{R}^{n}$ and controls $u \in \Omega \subset \mathbf{R}^{m}, 0 \in$ int $\Omega$ and with continuous function $f$ such that $f(0,0)=0$ and in each set $\left\{[x, u]\left|0<\rho_{1} \leq\right| x \mid \leq \rho_{2}, u \in \Omega\right\}$ the following Lipschitz condition holds:

$$
\left|f\left(x^{1}, u^{1}\right)-f\left(x^{2}, u^{2}\right)\right| \leq L_{1}\left(\rho_{1}, \rho_{2}\right)\left(\left|x^{1}-x^{2}\right|+\left|u^{1}-u^{2}\right|\right)
$$

Assume that there is a function $\theta(x)$ defined on $G=\{x|| x \mid \leq R\}$ with some $R>0$ and such that

1) $\theta(x)>0$, whenever $x \neq 0$ and $\theta(0)=0$;

2) $\theta(\cdot)$ is of class $C$ everywhere and of class $C^{1}$ everywhere except the origin $x=0$;

3) there exists $C>0$ such that the set $Q=\{x \mid \theta(x) \leq C\}$ is bounded and $Q \subset\{x|| x \mid<R\}$;

4) there exists a feedback $u(x)$ with $u(x) \in \Omega$, whenever $x \in Q$, and

$$
\frac{\partial \theta(x)}{\partial x} f(x, u(x))=\sum_{i=1}^{n} \frac{\partial \theta(x)}{\partial x_{i}} f_{i}(x, u(x)) \leq-\beta \theta^{1-\frac{1}{\alpha}}(x), \quad x \in Q \backslash\{0\}
$$

with some $\alpha>0, \beta>0$, in addition, in each set $K\left(\rho_{1}, \rho_{2}\right)=\left\{x \in \mathbf{R}^{n}\left|0<\rho_{1} \leq\right| x \mid \leq \rho_{2}\right\}$ the feedback $u(x)$ satisfies the following Lipschitz condition:

$$
\left|u\left(x^{1}\right)-u\left(x^{2}\right)\right| \leq L_{2}\left(\rho_{1}, \rho_{2}\right)\left|x^{1}-x^{2}\right| \quad \text { for all } \quad\left\{x^{1}, x^{2}\right\} \subset K\left(\rho_{1}, \rho_{2}\right) .
$$

Then, for each $x^{0} \in Q$ and each $t_{0} \in \mathbf{R}$, the trajectory, of the system $\dot{x}=f(x, u(x))$, defined by $x\left(t_{0}\right)=x^{0}$ satisfies $\lim _{t \rightarrow t_{0}+T} x(t)=0$ and $x(t)=0$ for all $t \geq t_{0}+T$ with some $T \leq\left(\frac{\alpha}{\beta}\right) \theta^{\frac{1}{\alpha}}\left(x^{0}\right) ;$ (note that if $\alpha=+\infty$ then $x(t) \rightarrow 0$ as $t \rightarrow+\infty$ ).

3. Main result. We consider the following control system:

$$
\begin{aligned}
& \dot{x}_{k}=f_{k}\left(x_{1}, \ldots, x_{k+1}\right), \quad k=1, \ldots, n-1, \\
& \dot{x}_{n}=f_{n}\left(x_{1}, \ldots, x_{n}, u\right)
\end{aligned}
$$

with controls $u \in \mathbf{R}^{m}=\mathbf{R}^{m_{n+1}}$, with states $x=\left[x_{1}, \ldots, x_{n}\right]^{T} \in \mathbf{R}^{m_{1}+\ldots+m_{n}}$, with $x_{i} \in \mathbf{R}^{m_{i}}$, $m_{i} \leq m_{i+1}$, and with $f_{i}\left(x_{1}, \ldots, x_{i+1}\right) \in \mathbf{R}^{m_{i}}$. We define 


$$
f(x, u)=\left(\begin{array}{c}
f_{1}\left(x_{1}, x_{2}\right) \\
f_{2}\left(x_{1}, x_{2}, x_{3}\right) \\
\ldots \ldots \\
f_{n}\left(x_{1}, \ldots, x_{n}, u\right)
\end{array}\right)
$$

and assume that system (2) satisfies the following conditions:

(I) $f \in C^{n+1}\left(\mathbf{R}^{m_{1}+\ldots+m_{n}} \times \mathbf{R}^{m} ; \mathbf{R}^{m_{1}+\ldots+m_{n}}\right)$,

(II) $f_{i}\left(x_{1}, \ldots, x_{i}, \mathbf{R}^{m_{i+1}}\right)=\mathbf{R}^{m_{i}}$ for every $\left[x_{1}, \ldots, x_{i}\right] \in \mathbf{R}^{m_{1}} \times \ldots \times \mathbf{R}^{m_{i}}$, and every $i=$ $=1, \ldots, n$,

(III) there exist $x_{i}^{*} \in \mathbf{R}^{m_{i}}, 1 \leq i \leq n$, and $u^{*}=x_{n+1}^{*}$ in $\mathbf{R}^{m_{n+1}}$ such that

$$
\operatorname{rank} \frac{\partial f_{i}}{\partial x_{i+1}}\left(x_{1}^{*}, \ldots, x_{i+1}^{*}\right)=m_{i} \text { for every } i=1, \ldots, n,
$$

and such that $f\left(x^{*}, u^{*}\right)=0$, where $x^{*}=\left[x_{1}^{*}, \ldots, x_{n}^{*}\right]$.

We want to study the problem of global finite-time stabilization of system (2) into $\left[x^{*}, u^{*}\right]$. This means that we are looking for a global feedback $u=u(t, x)$ which is of class $C^{1}$ everywhere except $\mathbf{R} \times\left\{x^{*}\right\}$ and uniformly bounded in $\mathbf{R} \times \overline{B_{\sigma}\left(x^{*}\right)}$ with some $\sigma>0$ and which satisfies the following conditions: (a) every solution of the closed-loop system $\dot{x}=f(x, u(t, x))$ given by any initial condition $x\left(t_{0}\right)=x^{0}$ converges to $x^{*}$ in finite time, i.e., there exists a finite $T\left(t_{0}, x^{0}\right)>0$ such that $x(t) \rightarrow x^{*}$ as $t \rightarrow t_{0}+T\left(t_{0}, x^{0}\right)$ and $x(t)=0$ for all $t \geq t_{0}+T\left(t_{0}, x^{0}\right)$ and (b) $x^{*}$ is a globally asymptotically stable equilibrium for the closed-loop system $\dot{x}=f(x, u(t, x))$. Our main result is as follows.

Theorem 3.1. Suppose that system (2) satisfies conditions (I)-(III). Then there exists the above-mentioned feedback $u=u(t, x)$ (at least T-periodic in time with any $T>0$ ) which solves the above-mentioned problem. Furthermore, in some neighborhood of $x^{*} \in \mathbf{R}^{m_{1}+\ldots+m_{n}}$, the feedback $u(t, x)$ is time-invariant, i.e., $u(t, x)=u(x)$ and it satisfies all the conditions 1)4) of Theorem 2.1 (along with (1)) everywhere in this neighborhood with some controllability function $\theta(x)$.

Given a feedback $u(t, x)$, any $t_{0} \in \mathbf{R}$, and any $x^{0}$ in $\mathbf{R}^{m_{1}+\ldots+m_{n}}$, let $t \mapsto x\left(t, t_{0}, x^{0}, u(\cdot, \cdot)\right)$ denote the trajectory, of system (2), defined by this feedback and by the initial condition $x\left(t_{0}\right)=$ $=x^{0}$ (in our argument, it will be clear from the context that the uniqueness of the solution of the corresponding Cauchy problem is provided).

First of all, let us explain why we are looking for a feedback of the form $u(t, x)$ although the dynamics of (2) does not depend on time. This is caused by the global properties of the "generalized triangular form" defined by conditions (I) - (III) and can be demonstrated by the following example proposed in $[14,18]$ for the classical stabilization problem (this is applied both to the problem of classical global asymptotic stabilization and to our synthesis problem).

Example 3.1. Consider the nonlinear system

$$
\begin{aligned}
& \dot{x}_{1}=b x_{2}^{3}-\left(1-a x_{1}^{2}\right) x_{2}, \\
& \dot{x}_{2}=u,
\end{aligned}
$$


$(a>0, b>0)$ which satisfies all the above-mentioned Conditions (I) - (III), and assume, for instance, that there is a feedback law $u=u\left(x_{1}, x_{2}\right)$ of class $C^{1}$, which globally asymptotically stabilizes (4) into $[0,0]$. Define $g(x)$ by $g(x):=\left[b x_{2}^{3}-\left(1-a x_{1}^{2}\right) x_{2}, u\left(x_{1}, x_{2}\right)\right]^{T}$, and $C$ by $C:=$ $:=\left\{\left[x_{1}, x_{2}\right] \in \mathbf{R}^{2} \mid a x_{1}^{2}+b x_{2}^{2}=1\right\}$. Since the feedback $u=u(x)$ is continuous on $C$, and globally stabilizes (4), we obtain $u(x) \neq 0$ for all $x \in C$. Then the map given by $C \ni x \mapsto$ $\mapsto \frac{g(x)}{|g(x)|}=\left[0, \frac{u(x)}{|u(x)|}\right]^{T}$ is well-defined. On the one hand its degree equals 0 , but on the other, there exists a homotopy between this map and the map given by $C \ni x \mapsto(-x) \in C$ (see the proof of the well-known Brockett necessary condition for stabilization [3], which can be also found in [22, p. 184]). This contradiction proves that there is no any feedback $u=u(x)$ of class $C^{1}$ which globally stabilizes (4). The same argument can be applied for the global synthesis problem considered in the current paper. (Note that (4) is even SISO (single-input and singleoutput), i.e., with scalar $x_{i}$ and $u$ ).

Our next example is concerned with the MIMO (multi-input and multi-output) case and with different $m_{i}$.

Example 3.2. Consider the following MIMO nonlinear system

$$
\begin{aligned}
& \dot{x}_{1}=\left(y_{3}^{2}-y_{1}^{2}-1\right) \cos y_{2}-x_{2}^{2}-1, \\
& \dot{x}_{2}=\left(y_{3}^{2}-y_{1}^{2}-1\right) \sin y_{2}-x_{1}^{2}-1, \\
& \dot{y}_{1}=\mathrm{e}^{y_{1}} u_{2}, \\
& \dot{y}_{2}=u_{3} \cos u_{1}-\frac{1}{x_{1}^{2}+1}, \\
& \dot{y}_{3}=u_{3} \sin u_{1}-\frac{1}{x_{2}^{2}+1},
\end{aligned}
$$

with states $\left[x_{1}, x_{2}, y_{1}, y_{2}, y_{3}\right]^{T} \in \mathbf{R}^{5}$ and controls $\left[u_{1}, u_{2}, u_{3}\right]^{T} \in \mathbf{R}^{3}$. System (5) has form (2) with $m_{1}=2, m_{2}=m_{3}=3$, and with $n=2$. It is clear that system (5) has many singular points (every point $\left[x_{1}, x_{2}, y_{1}, y_{2}, y_{3}\right]$ such that $y_{3}^{2}-y_{1}^{2}-1=0$ is singular). However, each equilibrium point satisfies the following conditions:

$$
\left(y_{3}^{2}-y_{1}^{2}-1\right)^{2}=\left(x_{2}^{2}+1\right)^{2}+\left(x_{1}^{2}+1\right)^{2}>0, \quad y_{1}^{2}+y_{3}^{2}>0,
$$

and

$$
u_{3}^{2}=\left(\frac{1}{x_{1}^{2}+1}\right)^{2}+\left(\frac{1}{x_{2}^{2}+1}\right)^{2}>0
$$

and, therefore, it is regular, i.e., satisfies condition (III). Finally, (5) satisfies conditions (I), (II), and, therefore, our Theorem 3.1 is applicable to system (5) for each equilibrium point of this system. 
4. Proof of Theorem 3.1. First, we rewrite system (2) in the following form:

$$
\begin{aligned}
& \dot{x}_{1}^{(1)}=f_{1}^{(1)}\left(x_{1}^{(1)}, x_{2}^{(1)}, x_{2}^{(2)}\right), \\
& \dot{x}_{k}^{(1)}=f_{k}^{(1)}\left(x_{1}^{(1)}, \ldots, x_{k}^{(1)}, \ldots, x_{k}^{(k)}, x_{k+1}^{(1)}, \ldots, x_{k+1}^{(k+1)}\right), \\
& \dot{x}_{k}^{(k)}=f_{k}^{(k)}\left(x_{1}^{(1)}, \ldots, x_{k}^{(1)}, \ldots, x_{k}^{(k)}, x_{k+1}^{(1)}, \ldots, x_{k+1}^{(k+1)}\right), \\
& \dot{x}_{n}^{(1)}=f_{n}^{(1)}\left(x_{1}^{(1)}, \ldots, x_{n}^{(1)}, \ldots, x_{n}^{(n)}, u^{(1)}, \ldots, u^{(n+1)}\right), \\
& \dot{x}_{n}^{(n)}=f_{n}^{(n)}\left(x_{1}^{(1)}, \ldots, x_{n}^{(1)}, \ldots, x_{n}^{(n)}, u^{(1)}, \ldots, u^{(n+1)}\right),
\end{aligned}
$$

where

$$
\begin{gathered}
x_{k}=\left[x_{k}^{(1)}, \ldots, x_{k}^{(k)}\right]^{T} \in \mathbf{R}^{m_{k}}, \quad f_{k}=\left[f_{k}^{(1)}, \ldots, f_{k}^{(k)}\right]^{T} \in \mathbf{R}^{m_{k}}, \quad k=1, \ldots, n, \\
u=\left[u^{(1)}, \ldots, u^{(n+1)}\right]^{T} \in \mathbf{R}^{m_{n+1}}
\end{gathered}
$$

with

$$
x_{i}^{(i)} \in \mathbf{R}^{m_{1}}, \quad f_{i}^{(i)} \in \mathbf{R}^{m_{1}}, \quad i=1, \ldots, n, \quad u^{(n+1)} \in \mathbf{R}^{m_{1}}, \quad u^{(1)} \in \mathbf{R}^{m_{n+1}-m_{n}}
$$

and

$$
x_{i}^{(j)} \in \mathbf{R}^{m_{i-j+1}-m_{i-j}}, \quad f_{i}^{(j)} \in \mathbf{R}^{m_{i-j+1}-m_{i-j}}, \quad 1 \leq j \leq i-1, \quad i=2, \ldots, n,
$$

and $x_{i}^{(j)}, f_{i}^{(j)}$ are defined by the following conditions:

$$
\operatorname{rank} \frac{\partial f_{k}^{(j)}\left(x_{1}^{*}, \ldots, x_{k+1}^{*}\right)}{\partial x_{k+1}^{(k+1)}}=m_{1}, \quad k=1, \ldots, n, \quad \operatorname{rank} \frac{\partial f_{n}^{(n)}\left(x^{*}, u^{*}\right)}{\partial u^{(n+1)}}=m_{1},
$$

$$
\operatorname{rank} \frac{\partial\left(f_{k}^{(1)}, \ldots, f_{k}^{(k)}\right)\left(x^{*}\right)}{\partial\left(x_{k+1}^{(2)}, \ldots, x_{k+1}^{(k+1)}\right)}=m_{k}, \quad k=1, \ldots, n, \quad \operatorname{rank} \frac{\partial f_{n}^{(n)}\left(x^{*}, u^{*}\right)}{\partial\left(u^{2}, \ldots, u^{(n+1)}\right)}=m_{n}
$$

Let us note that if $m_{i-j}=m_{i-j+1}$, then $x_{i}^{(j)}$ is omitted (its dimension equals zero); in particular, for $m_{1}=\ldots=m_{n}=m_{n+1}$, we have $x_{k}=x_{k}^{(k)} \in \mathbf{R}^{m_{k}}=\mathbf{R}^{m_{n+1}}, k=1, \ldots, n$, $u=u^{(n+1)} \in \mathbf{R}^{m_{n+1}}$. 
Then we consider the following transformation of states and controls (note that this is a natural extension of the transformation considered in [9] for the special case $m_{1}=\ldots$ $\left.\ldots=m_{n+1}=1\right)$ :

$$
\begin{aligned}
\xi_{1}^{(1)} & =F_{1}^{(1)}\left(x_{1}\right):=x_{1}^{(1)} \\
\xi_{k+1}^{(1)} & =F_{k+1}^{(1)}\left(x_{1}, \ldots, x_{k}, x_{k+1}\right):=x_{k+1}^{(1)} \\
\xi_{k+1}^{(l)} & =F_{k+1}^{(l)}\left(x_{1}, \ldots, x_{k}, x_{k+1}\right):= \\
& :=\sum_{i=1}^{k} \sum_{j=1}^{i} \frac{\partial F_{k}^{(l-1)}}{\partial x_{i}^{(j)}} f_{i}^{(j)}\left(x_{1}, \ldots, x_{i+1}\right), \quad \text { for } \quad 2 \leq l \leq k+1, \quad 1 \leq k \leq n-1, \\
v^{(1)} & =F_{n+1}^{(1)}(x, u):=u^{(1)}, \\
v^{(l)} & =F_{n+1}^{(l)}(x, u):=\sum_{i=1}^{n-1} \sum_{j=1}^{i} \frac{\partial F_{n}^{(l-1)}}{\partial x_{i}^{(j)}} f_{i}^{(j)}(x, u)+\sum_{j=1}^{n} \frac{\partial F_{n}^{(l-1)}}{\partial x_{n}^{(j)}} f_{n}^{(j)}(x, u), \quad l=2, \ldots, n+1 .
\end{aligned}
$$

It is easy to show that (8) is a local diffeomorphism around $\left[x^{*}, u^{*}\right]$ and (in some neighborhood of $\left[x^{*}, u^{*}\right]$ ) maps diffeomorphically the trajectories of system (6) (i.e., system (2)) onto the trajectories of the following linear system:

$$
\begin{aligned}
& \dot{\xi}_{k+i-1}^{(i)}=\xi_{k+i}^{(i+1)}, \quad i=1, \ldots, n-k, \quad \dot{\xi}_{n}^{(n-k+1)}=v^{(n-k+2)}, \quad k=1, \ldots, n-1, \\
& \dot{\xi}_{n}^{(1)}=v^{(2)} .
\end{aligned}
$$

Rewrite system (9) in the following form:

$$
\dot{\xi}=A \xi+B v
$$

where $A$ and $B$ are the corresponding matrices defined by system (9). (It is clear that (10) is globally controllable.)

According to the result of [11] (see also [12, p. 121-139]), there exist some $a_{0}>0$ and some neighborhood of 0 such that the equation

$$
2 a_{0} \theta-\left\langle N^{-1}(\theta) \xi, \xi\right\rangle=0
$$

with

$$
N(\theta):=\int_{0}^{+\infty} e^{-\frac{t}{\theta}} e^{-A t} B B^{*} e^{-A^{*} t} d t
$$

has a unique solution for each $\xi \in B_{r}(0) \backslash\{0\}$ (for some small $r>0$ ) and defines a positive definite function $\theta(\xi)$ of class $C^{1}$ on $B_{r}(0) \backslash\{0\}$ and of class $C$ on $B_{r}(0)$ (for $\xi=0, \theta(0)=0$ by the definition of $\theta(\xi))$ and such that the control

$$
w(\xi):= \begin{cases}-B^{*} N^{-1}(\theta(\xi)) \xi & \text { if } \xi \in B_{r}(0) \backslash\{0\} \\ 0 & \text { if } \xi=0\end{cases}
$$


with this controllability function satisfies all the conditions 1) -4) of Theorem 2.1 with some $\alpha>0, \beta>0$, and, therefore (locally, around the origin), resolves the synthesis problem in the sense of Theorem 2.1. Furthermore ([12, p. 127] or [11]), for any $d>0$, it is possible to find some $a_{0}>0$ such that $|w(\xi)| \leq d$, whenever $\xi \in B_{r}(0)$.

Let $x=\Phi(\xi), u=\Psi(\xi, v)$ be a diffeomorphism which is inverse to the map given by (8) and which (locally, around the origin) maps the trajectories of system (10) onto the trajectories of system (2). Define

$$
\hat{u}(x):=\Psi(F(x), w(F(x))) \quad \text { and } \quad \Theta(x)=\theta(F(x))
$$

where $F(x)$ is the transformation of coordinates defined in (8) which (along with the transformation of controls given by (8)) brings (2) (or (6)) to (10).

Then, in some open neighborhood of $x^{*}$, we obtain

$$
\frac{\partial \Theta(x)}{\partial x} f(x, \hat{u}(x)) \leq-\frac{\beta}{\alpha} \Theta^{1-\frac{1}{\alpha}}(x), \quad \text { for all } \quad x \neq x^{*} \text { such that } \Theta(x) \leq \rho_{0}
$$

with some $\rho_{0}>0$. On the other hand, using the result of [18], we obtain that for any $T>0$, there exist $R_{0}>0$ and some $T$-periodic feedback $v(t, x)$ of class $C^{1}$, which globally asymptotically stabilizes system (2), and there is a $T$-periodic Lyapunov function $V(t, x)$ of class $C^{1}$ defined in some neighborhood of $\mathbf{R} \times\left\{x^{*}\right\}$ such that $V$ is positive definite $(V \geq 0$ and $V(t, x)=0$ if and only if $\left.x=x^{*}\right) ; V$ has the form

$$
V(t, x)=\left|x_{1}-x_{1}^{*}\right|^{2}+\left|x_{2}-\alpha_{1}\left(t, x_{1}\right)\right|^{2}+\ldots+\left|x_{n}-\alpha_{n-1}\left(t, x_{1}, \ldots, x_{n-1}\right)\right|^{2}
$$

with $\alpha_{i}\left(t, x_{1}^{*}, \ldots, x_{i}^{*}\right)=x_{i+1}^{*} \in \mathbf{R}^{m_{i+1}}$ and $\alpha_{i}\left(t+T, x_{1}, \ldots, x_{i}\right)=\alpha_{i}\left(t, x_{1}, \ldots, x_{i}\right)$ for all $t\left(\alpha_{i}\right.$ being of class $\left.C^{1}\right)$ and such that

$$
\frac{\partial V}{\partial t}+\frac{\partial V}{\partial x} f(x, v(t, x)) \leq-V(t, x) \text { for all }[t, x] \text { such that } V(t, x) \leq R_{0}
$$

Remark 1. Let us note that, since (2) is time-independent, the argument from [18] actually yields that $V(t, x)$ and $v(t, x)$ are also time-independent locally in some neighborhood of $\mathbf{R} \times$ $\times\left\{x^{*}\right\}$ (whereas $v(t, x)$ is time-varying and $T$-periodic globally, in general), therefore, without loss of generality we may assume that $V(t, x)=V(x), v(t, x)=v(x)$ and $\alpha_{i}\left(t, x_{1}, \ldots, x_{i}\right)=$ $=\alpha_{i}\left(x_{1}, \ldots, x_{i}\right)$ for all $[t, x]$ such that $V(t, x) \leq R_{0}$.

Fix any $R$ in $] 0, R_{0}[$. For any $c>0$, denote:

$$
\begin{gathered}
\Gamma_{c}:=\mathbf{R} \times\left\{x \in \mathbf{R}^{m_{1}+\ldots+m_{n}} \mid \Theta(x) \leq c\right\}, \\
\Lambda_{c}:=\left\{[t, x] \in \mathbf{R} \times \mathbf{R}^{m_{1}+\ldots+m_{n}} \mid V(t, x)<c\right\} .
\end{gathered}
$$

Remark 2. It is straightforward that both $\Gamma_{c}$ (see [12, p. 122] as well as $\left.[1,10,11]\right)$ and $\Lambda_{c}$ (see the form of $V(t, x))$ have the following properties: $\Gamma_{\hat{\rho}}$ and $\Lambda_{\hat{R}}$ are bounded for all $\hat{\rho} \in\left[0, \rho_{0}\right]$ and $\hat{R} \in\left[0, R_{0}\right]$, respectively, and $\sup _{[t, x] \in \Gamma_{c}}\left|x-x^{*}\right| \rightarrow 0$ and $\sup _{[t, x] \in \Lambda_{c}}\left|x-x^{*}\right| \rightarrow 0$ as $c \rightarrow+0$.

Take any small $\varepsilon_{0}>0$ and any small $\left.\rho \in\right] 0, \rho_{0}\left[\right.$ such that $\Gamma_{\rho+\varepsilon_{0}} \subset \Lambda_{R}$. 
Given any $\varepsilon \in] 0, \varepsilon_{0}\left[\right.$, take some $T$-periodic function $\lambda(t, x)$ of class $C^{\infty}\left(\mathbf{R} \times \mathbf{R}^{m_{1}+\ldots+m_{n}}\right.$; $[0,1])$ such that $\lambda(t, x)=1$ if $[t, x] \in \Gamma_{\rho} ; \lambda(t, x)=0$ if $[t, x] \notin \Gamma_{\rho+\varepsilon}$. Let $u_{\varepsilon}(t, x)$ of class $C\left(\mathbf{R} \times \mathbf{R}^{m_{1}+\ldots+m_{n}} ; \mathbf{R}^{m_{n+1}}\right) \bigcap C^{1}\left(\mathbf{R} \times\left(\mathbf{R}^{m_{1}+\ldots+m_{n}} \backslash\left\{x^{*}\right\}\right) ; \mathbf{R}^{m_{n+1}}\right)$ be given by

$$
u_{\varepsilon}(t, x)=\lambda(t, x) \hat{u}(x)+(1-\lambda(t, x)) v(t, x) .
$$

(Note that, although $\hat{u}(x)$ was defined locally around $x^{*}$ only, $u_{\varepsilon}(t, x)$ is well-defined everywhere because, if $[t, x] \notin \Gamma_{\rho+\varepsilon_{0}}$, then $\lambda(t, x)=0$, i. e., the first term is vanishing.) It is clear that for every $\left[t_{0}, x^{0}\right] \in \mathbf{R} \times \mathbf{R}^{m_{1}+\ldots+m_{n}}$ we obtain:

1) if $\left[t_{0}, x_{0}\right] \in \Gamma_{\rho}$, then $\left[t, x\left(t, t_{0}, x^{0}, u_{\varepsilon}(\cdot, \cdot)\right)\right] \in \Gamma_{\rho}$ for all $t \geq t_{0}$ and there is $T\left(t_{0}, x^{0}\right)>t_{0}$ such that $x\left(t, t_{0}, x^{0}, u_{\varepsilon}(\cdot, \cdot)\right) \rightarrow x^{*}$ as $t \rightarrow T\left(t_{0}, x^{0}\right)$ (furthermore, along such a trajectory, the inequality (11) holds);

2) if $\left[t_{0}, x_{0}\right] \in \Lambda_{R}$, then $\left[t, x\left(t, t_{0}, x^{0}, u_{\varepsilon}(\cdot, \cdot)\right)\right] \in \Lambda_{R}$ for all $t \geq t_{0}$;

3) for every $\left[t_{0}, x^{0}\right] \in \mathbf{R} \times \mathbf{R}^{m_{1}+\ldots+m_{n}}$ there exists $t_{1} \geq t_{0}$ such that $\left[t, x\left(t, t_{0}, x^{0}, u_{\varepsilon}(\cdot, \cdot)\right)\right] \in$ $\in \Lambda_{R}$ for all $t \geq t_{1}$.

Take any $\sigma>0$ such that $\Lambda_{\sigma+\delta} \subset \Gamma_{\rho}$ for some small $\delta>0$. Then, there are some $n \in \mathbf{N}$ and some $m \in \mathbf{N}$ such that for any $\left[t_{0}, x_{0}\right] \in \Lambda_{R}$ we have $\left[t, x\left(t, t_{0}, x^{0}, v(\cdot, \cdot)\right)\right] \in \Lambda_{\sigma}$ for all $t \geq t_{0}+n T$ and for any $\left[t_{0}, x_{0}\right] \in \Gamma_{\rho}$ we have $x\left(t, t_{0}, x^{0}, \hat{u}(\cdot)\right)=x^{*}$ for all $t \geq t_{0}+m T$.

Given $h \in] 0, \frac{T}{2}\left[\right.$, find any $(m+n+2) T$-periodic function $\mu_{h}(t)$ of class $C^{\infty}(\mathbf{R} ;[0,1])$ such that

$$
\mu_{h}(t)=\left\{\begin{array}{l}
0 \quad \text { if } \quad k(m+n+2) T+h \leq t \leq(k+1)(n+1) T+k(m+1) T-h, \\
1 \quad \text { if } \quad(k+1)(n+1) T+k(m+1) T+h \leq t \leq(k+1)(m+n+2) T-h
\end{array}\right.
$$

for all $k \in \mathbf{Z}$, and define

$$
\hat{u}_{h}(t, x):=\mu_{h}(t) u_{\varepsilon}(t, x)+\left(1-\mu_{h}(t)\right) v(t, x) .
$$

Then it is straightforward to check that if $h \in] 0, \frac{T}{2}\left[\right.$ is small enough, then the feedback $\hat{u}_{h}(t, x)$ resolves our problem of global stabilization in finite time and satisfies Theorem 3.1.

Acknowledgement. This work was performed during April-May 2008 while the second author was visiting Institute of Mathematics and Informatics, Ernst-Moritz-Arndt University of Greifswald. Svyatoslav Pavlichkov is grateful for the warm hospitality provided by Professor Werner H. Schmidt.

1. Bessonov G. A., Korobov V.I., Sklyar G. M. The problem of the stable synthesis of bounded controls for a certain class of non-steady systems // J. Appl. Math. and Mech. - 1988. - 52. - P. 11-17.

2. Bhat S. P., Bernstein D.S. Continuous finite-time stabilization of the translational and rotational double integrators // IEEE Trans. Automat. Control. - 1998. - 43. - P. 678-682.

3. Brockett R. Asymptotic stability and feedback stabilization // Different. Geometric Control Theory / Eds R.W. Brockett, R. S. Millman, and H. J. Sussmann. - Boston: Birkhäuser, 1983. - P. 181 - 191.

4. Celikovsky S., Nijmeijer H. Equivalence of nonlinear systems to triangular form: the singular case // Systems and Control Lett. - 1996. - 27. - P. 135-144.

5. Celikovsky S., Aranda-Bricaire E. Constructive nonsmooth stabilization of triangular systems // Systems and Control Lett. - 1999. - 36. - P. $21-37$. 
6. Gorr G. V., Ilyuhin A. A., Kovalev A. M., Savchenko A. Ya. Nonlinear analysis of the behavior of nonlinear systems. - Kiev: Naukova Dumka, 1984 (in Russian).

7. Huang X., Lin W., Yang B. Global finite-time stabilization of a class of uncertain nonlinear systems // Automatica. - 2005. - 41. - P. $881-888$.

8. Hong Y., Jiang Zh.-P., Feng G. Finite-time input-to-state stability and applications to finite-time control design // SIAM J. Contr. and Optim. - 2010. - 48. - P. 4395 - 4418.

9. Korobov V. I. Controllability and stability of some nonlinear systems // Differenc. Uravneniya. - 1973. - 9. - P. $614-619$.

10. Korobov V.I. A general approach to the solution of the synthesis problem by means of bounded controls // Mat. Sbornik. - 1979. - 109. - P. 582-606.

11. Korobov V.I., Sklyar G. M. Methods of constructing positionals controls and acceptable maximum principle // Differenc. Uravneniya. - 1990. - 26. - P. 1914-1924.

12. Korobov V.I. Controllability function method (Regular and chaotic dynamics). - Moscow; Izhevsk, 2007.

13. Korobov V. I., Pavlichkov S. S., Schmidt W. H. Global robust controllability of the triangular integro-differential Volterra systems // J. Math. Anal. and Appl. - 2005. - 309. - P. 743 - 760.

14. Korobov V. I., Pavlichkov S. S. Global properties of the triangular systems in the singular case // J. Math. Anal. and Appl. - 2008. - 342. - P. 1426-1439.

15. Kovalev A. M., Scherbak V.F. Controllability, observability and identification of dynamical systems. - Kyiv: Naukova Dumka, 1993 (in Russian).

16. Moulaya E., Perruquettib W. Finite time stability and stabilization of a class of continuous systems // J. Math. Anal. and Appl. - 2006. - 323. - P. 1430-1443.

17. Nam K., Arapostathis A. A model reference adaptive control scheme for pure-feedback nonlinear systems // IEEE Trans. Automat. Control. - 1988. - 33. - P. 803-811.

18. Pavlichkov S. S., Ge S. S. Global stabilization of the generalized MIMO triangular systems with singular inputoutput links // IEEE Trans. Automat. Control. - 2009. - 54(8). - P. $1794-1806$.

19. Respondek W. Global aspects of linearization, equivalence to polynomial forms and decomposition of nonlinear control systems // Algebraic and Geom. Meth. in Nonlinear Contr. Theory / Eds M. Fliess, M. Hazewinkel. - Dordrecht: Reidel, 1986. - P. 257-284.

20. Saberi A., Kokotovic P. V., Sussmann H. J. Global stabilization of partially linear composite systems // SIAM J. Contr. Optimiz. - 1990. - 28, № 6. - P. 1491-1503.

21. Sklyar G. M., Sklyar K. V., Ignatovich S. Yu. On the extension of the Korobov's class of linearizable triangular systems by nonlinear control systems of the class $C^{1}$ // Syst. Contr. Lett. - 2005. - 54. - P. 1097-1108.

22. Sontag E. D. Mathematical control theory: deterministic finite dimensional systems. - New York: Springer, 1990.

23. Tsinias $J$. A theorem on global stabilization of nonlinear systems by linear feedback // Syst. Contr. Lett. 1991. - 17. - P. 357-362.

24. Tsinias J. Partial-state global stabilization for general triangular systems. - Syst. Contr. Lett. - 1995 . - 24. - P. 139-145.

25. Tsinias J. Triangular systems: A global extension of the Coron-Praly theorem on the existence of feedbackintegrator stabilisers // Eur. J. Contr. - 1997. - 3, № 1. - P. 37 - 46.

Received 18.05.10,

after revision -15.11 .11$ 